\title{
BMJ Open Quality Implementing the WHO Safe Childlbirth Checklist: lessons learnt on a quality improvement initiative to improve mother and newborn care at Gobabis District Hospital, Namibia
}

\author{
Leonard Kabongo, ${ }^{1}$ Jonathon Gass, ${ }^{2}$ Beatrice Kivondo, ${ }^{1}$ Nabihah Kara, ${ }^{2}$ \\ Katherine Semrau, ${ }^{2,3}$ Lisa R Hirschhorn ${ }^{4}$
}

To cite: Kabongo L, Gass J, Kivondo B, et al. Implementing the WHO Safe Childbirth Checklist: lessons learnt on a quality improvement initiative to improve mother and newborn care at Gobabis District Hospital, Namibia.BMJ Open Quality 2017;6:e000145. doi:10.1136/ bmjoq-2017-000145

- Additional material is published online only. To view, please visit the journal online (http://dx.doi.org/10.1136/ bmjoq-2017-000145).

Received 18 June 2017 Accepted 22 June 2017

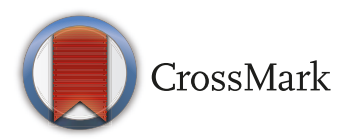

${ }^{1}$ Gobabis Hospital, Ministry of Health and Social Services, Gobabis, Namibia

${ }^{2}$ Ariadne Labs, Harvard T.H. Chan School of Public Health and Brigham \& Women's Hospital, Boston, Massachusetts USA

${ }^{3}$ Division Global Health Equity, Brigham and Women's Hospital, Boston, Massachusetts, USA ${ }^{4}$ Feinberg School of Medicine, Northwestern University, Chicago, Illinois, USA

Correspondence to Dr Leonard Kabongo, Gobabis Hospital, Ministry of Health and Social Services, Gobabis, Namibia; leonkab1@yahoo.com

\section{ABSTRACT}

Background Although there are many evidence-based practices that reduce the risk of maternal and neonatal mortality around the time of birth, there remains a gap between what is known and the care received. This knowdo gap is a source of preventable maternal and perinatal deaths and is the focus of improvement efforts in many countries. Following an increase in perinatal and maternal deaths, Gobabis District Hospital initiated a quality improvement $(\mathrm{Ql})$ initiative to increase adherence to these WHO Safe Childbirth Checklist (SCC)-targeted essential birth practices (EBPs).

Methods We implemented the SCC with support from leadership, coaching and organisational redesign. Implementation was led by a facility champion supported by a QI team and adapted through a series of three 8-week Plan-Do-Study-Act (PDSA) cycles.

Results During the 6-month period, we observed an improvement of average EBPs delivered from $68 \%$ to $95 \%$. We also found reductions in perinatal mortality rates from 22 deaths/1000 deliveries to $13.8 / 1000$ deliveries largely due to a drop in fresh stillbirths.

Conclusion We conclude that replicating the programme is feasible, acceptable and effective in areas where gaps exist, but it requires local leadership, ongoing coaching and adaptation through PDSA cycles.

\section{PROBLEM}

Namibia is a country in southwestern Africa with a maternal mortality ratio in 2013 estimated at 385/100000 live births compared with 249/100000 in 2000 and a neonatal mortality rate at 20/1000 live births versus $25 / 1000$ in $2000 .{ }^{1}$ These data suggest that the country had encountered challenges in meeting Millennium Development Goals (MDGs) 4 and 5. At Gobabis District Hospital, located in eastern Namibia, data from 2014 showed a maternal mortality ratio of 163/100 000 live births and perinatal death rate, defined as fresh stillbirths plus early neonatal deaths ( $<7$ days) of 26/1000 live births, and an early neonatal mortality rate of $17 / 1000$ live births. ${ }^{2}$ Haemorrhage and complications of abortions were the major cause of maternal deaths, and severe prematurity and birth asphyxia were the leading causes of perinatal deaths. ${ }^{3}$

Here, we describe the successful implementation of the WHO Safe Childbirth Checklist (SCC) to improve adherence to essential birth practices (EBPs) and to address some of the underlying causes of mortality among mothers delivering at the hospital and their newborns.

\section{BACKGROUND}

In 2000, the WHO introduced the MDGs to tackle different threats to the global population. Goals 4 and 5 specifically focused on maternal and child health. Member states agreed to reduce maternal mortality ratios by three-quarters and neonatal mortality rates by two-thirds from 1990 to $2015 .{ }^{4}$ EBPs are a core set of practices that are proven to reduce maternal and newborn harm by preventing or managing the major global causes of maternal deaths (haemorrhage, hypertensive disorders, infections and obstructed labour) and neonatal deaths (birth asphyxia, birth trauma, prematurity and infections). ${ }^{5}$ However, inconsistent adherence to EBPs is widely described and represents a risk to maternal and newborn safety. In response, the WHO, in collaboration with Ariadne Labs and a range of stakeholders, designed the SCC, which includes 29 EBPs known to reduce maternal and newborn morbidity and mortality organised into four pause points: on admission, before pushing (or caesarean section), within 1 hour after delivery and before discharge. ${ }^{56}$ Each task on the Checklist is a crucial action that, if missed, can result in severe harm for the mother, the newborn or both. 
Initial pilot testing at a single site in Karnataka, India, found that introduction of the checklist supported by coaching and leadership commitment resulted in significant improvements in EBP adherence from an average of 10 of 29 practices at baseline (95\% CI 9.4 to 10.1) to an average of 25 of 29 practices after SCC implementation (95\% CI 24.6 to $25.3, \mathrm{p}<0.001){ }^{7}$ Since then, the checklist was also made available through the WHO accompanied by an implementation guide to support facilities and organisations interested in further spread and evaluation. ${ }^{8}$ Based on experience that simply mandating a checklist or supplying it alone will not result in behavioural change, the guide describes a coaching-supported approach, which includes active leadership engagement. A cross-sectional study from Sri Lanka found that the SCC was both feasible and acceptable and adherence to EBPs during use was 21 of 29 (range 15\% to $89 \%$ provided per delivery depending on the EBP) to show impact on improving quality. ${ }^{9}$ Similar high uptake has been described in India with higher EBP delivery compared with facilities not targeted for SCC implementation. ${ }^{10}$

\section{DESIGN}

Gobabis District Hospital is the only District Hospital in the Omaheke Region in eastern Namibia serving a population of 72223 with a population density as low as 0.86 / $\mathrm{km}^{2}$. Gobabis District Hospital is equipped with 172 beds, including 20 beds in the maternity ward, with caesarean section capability. Maternity care occurs in the antenatal care ward, postnatal care ward and two delivery rooms with 2200 deliveries annually. Eight registered nurse midwives and two doctors staff the maternity ward in alternating shifts.

Based on familiarity with the WHO Safe Surgical Checklist, the Gobabis District hospital leadership decided to test the implementation of the WHO SCC supported by coaching conducted by maternity ward leaders to reduce preventable maternal and neonatal deaths. The aim was to improve adherence to EBPs through the use of the WHO SCC for all facility-based deliveries and to reduce preventable in-hospital perinatal mortality, specifically fresh stillbirths and early neonatal in-hospital deaths.

\section{STRATEGY}

A quality improvement (QI) team was convened to develop and test an intervention to reduce maternal and perinatal mortality. Members included one doctor and six nurse-midwives. The team employed the Model for Improvement and planned for rapid cycle testing using the Plan-Do-Study-Act (PDSA) approach. ${ }^{11}$ This work built on existing QI capacity at the hospital from work in HIV/AIDS. Based on the discussion of potential root causes, the team identified that despite relevant trainings, including emergency obstetric care and life-saving skills done at least two times per year, poor adherence to EBPs persisted as a primary driver. Secondary drivers included frequent staff changes in maternity and the need for leadership to provide stronger motivation and support for the changes necessary to ensure quality.

\section{MEASUREMENT}

Data from all checklists used in the maternity ward were entered into a secure Microsoft Excel database. Deliveries for which a checklist was not filled out were excluded from analysis of EBP adherence analysis. In-hospital mortality data were extracted from facility-based registers. Fresh stillbirths included babies born with no sign of life with gestation age of 26 weeks and above for mothers admitted for labour and delivery. Early neonatal in-hospital deaths included all newborns born alive that died before discharge from the hospital and within 7 days of birth. Perinatal deaths included fresh stillbirths and early newborn in-hospital deaths. Early in-hospital neonatal mortality rates were reported by the number of deaths per 1000 live births; fresh stillbirth and perinatal mortality rates were reported by the number of fresh stillbirth or deaths per 1000 live births plus fresh stillbirths, respectively.

\section{RESULTS}

PDSA cycle 1

Plan

Initial steps included adaptation of the SCC checklist, formal introduction of the WHO SCC to staff and a plan for ongoing support through coaching and performance feedback by hospital leadership.

Do

The intervention started the week of 17 February 2015 with introduction of the WHO SCC and initial data collection and review completed as planned.

\section{Adaptation of the WHO SCC}

The WHO SCC was modified for the local context by the midwives and doctors in Gobabis District Hospital during two sets of grand rounds (online supplementary appendix 1). Following the existing WHO SCC format, the checklist implemented included 29 EBPs, organised into the 4 pause points. Essential practices included prompt referral for high-risk pregnancies, hand washing, management of partograph, administering magnesium sulfate or giving antibiotics to the mother or her baby when needed, preparing essential obstetric supplies before delivery, administering oxytocin immediately postpartum and giving health education on danger signs, offering family planning and ensuring neonatal immunisation prior to discharge. Adaptations included clarification of referral process within the hospital and identification of referral into the facility, recommendation of delayed cord clamping, adherence to antiretroviral drugs for prevention of mother-to-child transmission if the mother was identified as HIV positive and use of antibiotics for the mother and child in cases where the woman delivered outside the hospital (ie, at home or on the way to the 
facility). Additional modifications focused on taking of maternal temperature, blood pressure and haemoglobin prior to discharge, as well as appropriate family planning methods.

\section{Introduction of the WHO SCC}

The adapted SCC was introduced to the maternity ward staff during a training that demonstrated its use along with neonatal resuscitation training. The expectation was that the birth attendants would use the SCC as a recall and documentation tool for the delivery of the EBPs.

\section{Follow-up support of WHO SCC use}

Doctors coached birth attendants weekly with monitoring and evaluation of adherence to the WHO SCC elements and feedback to enhance motivation. Every week, checklists were collected and data captured in an excel data sheet to capture the number of checklist used, the number of deliveries, the EBPs completed and maternal and newborn deaths. These data were reviewed weekly with the QI team and were used to inform the current and subsequent PDSA cycles.

\section{Study}

To estimate the baseline data on birthing practices by midwives, we observed their behaviours during all pause points for the first week using the WHO SCC. The checklist was available during this baseline data collection but had not been formally introduced or supported. Eight deliveries were observed with an average of only $68 \%$ of EBPs completed by the midwives and $75 \%$ of medications and supplies available over the week (figures 1 and 2).

From weeks 2 to 8 , we introduced the WHO SCC as planned and observed EBPs adherence among midwives using the checklist. Following the first PDSA cycle of 8 weeks, checklist use remained low, with usage in only $43 \%$ of deliveries. On average, $75 \%$ of EBPs were completed, and the EBPs that were least often conducted included having a birth companion present and checking for danger signs after delivery.

\section{Act}

The QI team met to review and discuss possible root causes for the lack of improvement and solutions. Identified challenges included that the staff saw the WHO SCC as an additional burden in paperwork rather than a tool to help them provide better care; further, checklists were sometimes completed and then lost. Staff rotation was another factor that resulted in new staff unfamiliar with the checklist. Also, there was a need to change the coaching from physician-led to peer-to-peer (midwife-tomidwife) coaching to increase birth attendant ownership and frequency.

\section{PDSA cycle 2}

Plan

We designed and implemented a stronger coaching model to support new staff knowledge and uptake of the checklist. The changes included a transition from a doctor-to-nurse coaching model to peer-to-peer coaching with two staff nurses identified as QI champions. Coaching capacity among the champions was enhanced through on-the-job training in coaching in QI by two hospital leaders, one who had previous training and experience in QI coaching and one who is a national QI trainer. The WHO SCC orientation was also strengthened to include more adult learning principles, including role-plays and games to increase ownership and acceptability and make the training more engaging. Another change was inclusion of the front-line nurses in the ongoing weekly data feedback sessions on EBP data to develop self-confidence, engage in ongoing improvement

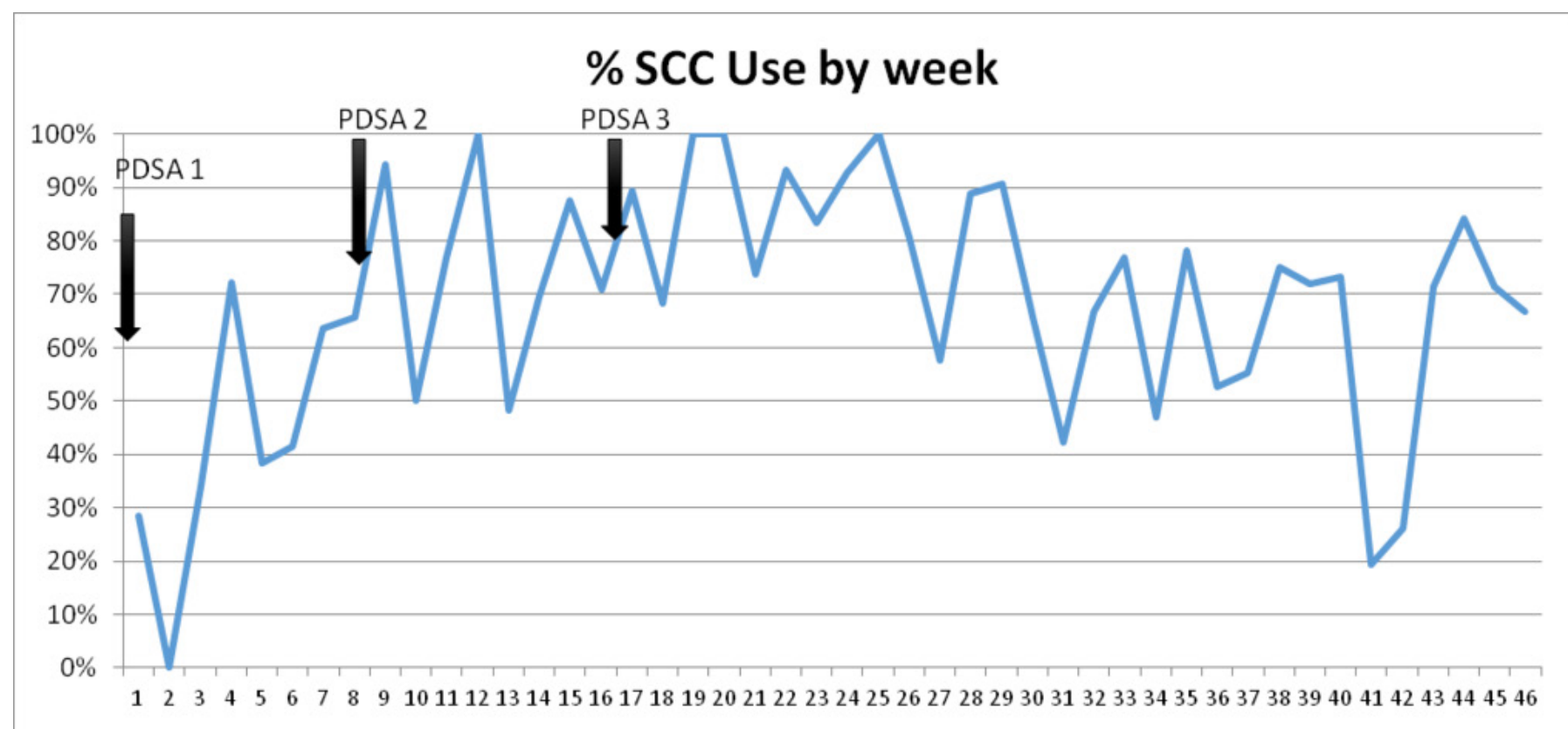

Figure 1 SCC use by week in Gobabis District Hospital, Namibia. PDSA, Plan-Do-Study-Act; SCC, Safe Childbirth Checklist. 


\section{Percent of EBPs completed by week}

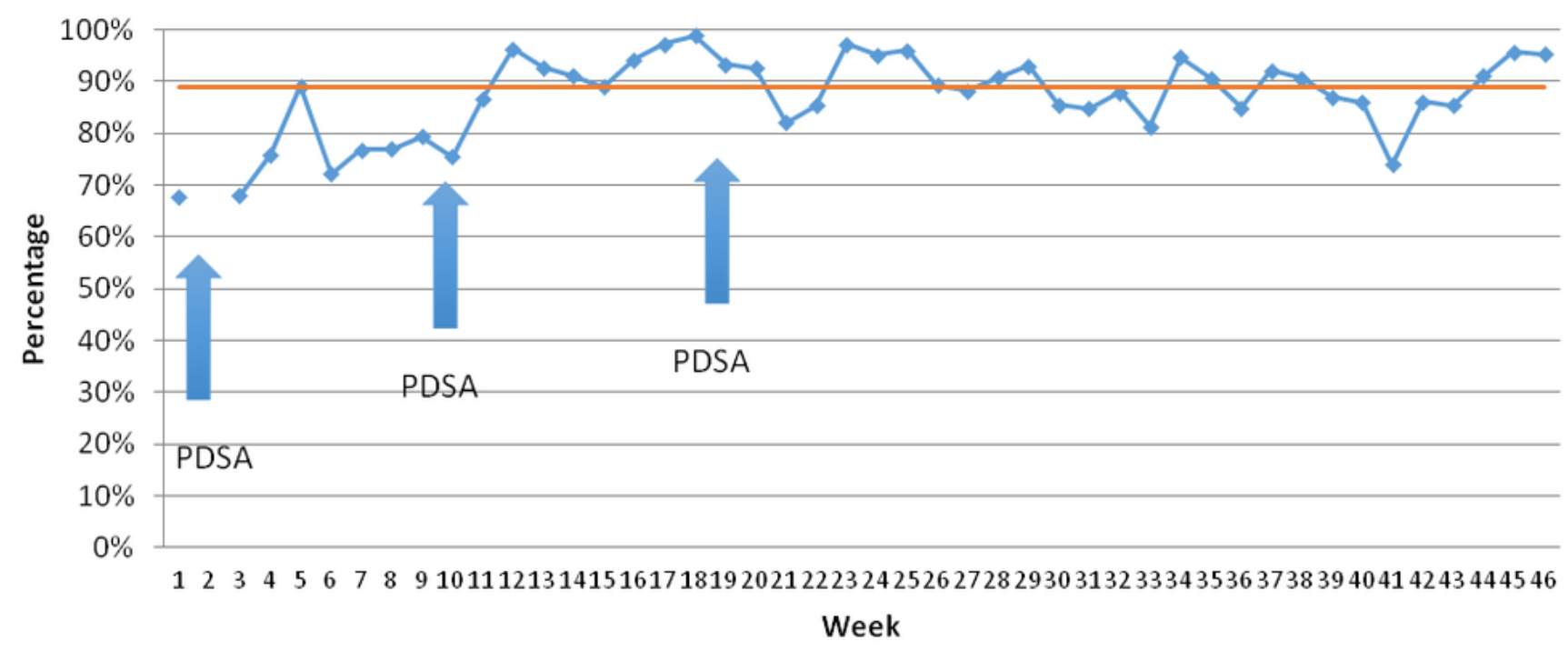

Median

Figure 2 Change in average number of EBPs completed by week and median overall. Note: no Safe Childbirth Checklists were completed in week 2. EBPs, essential birth practices; PDSA, Plan-Do-Study-Act.

and increase the opportunity for ongoing learning and Do input into improvements. To avoid lost checklists that had been completed, the completed checklist was stapled to the maternity record before being sent to the record room.
From weeks 9 to 16, engagement, support and regular feedback on behavioural adherence were provided as planned, with ongoing data collection, review and feedback.

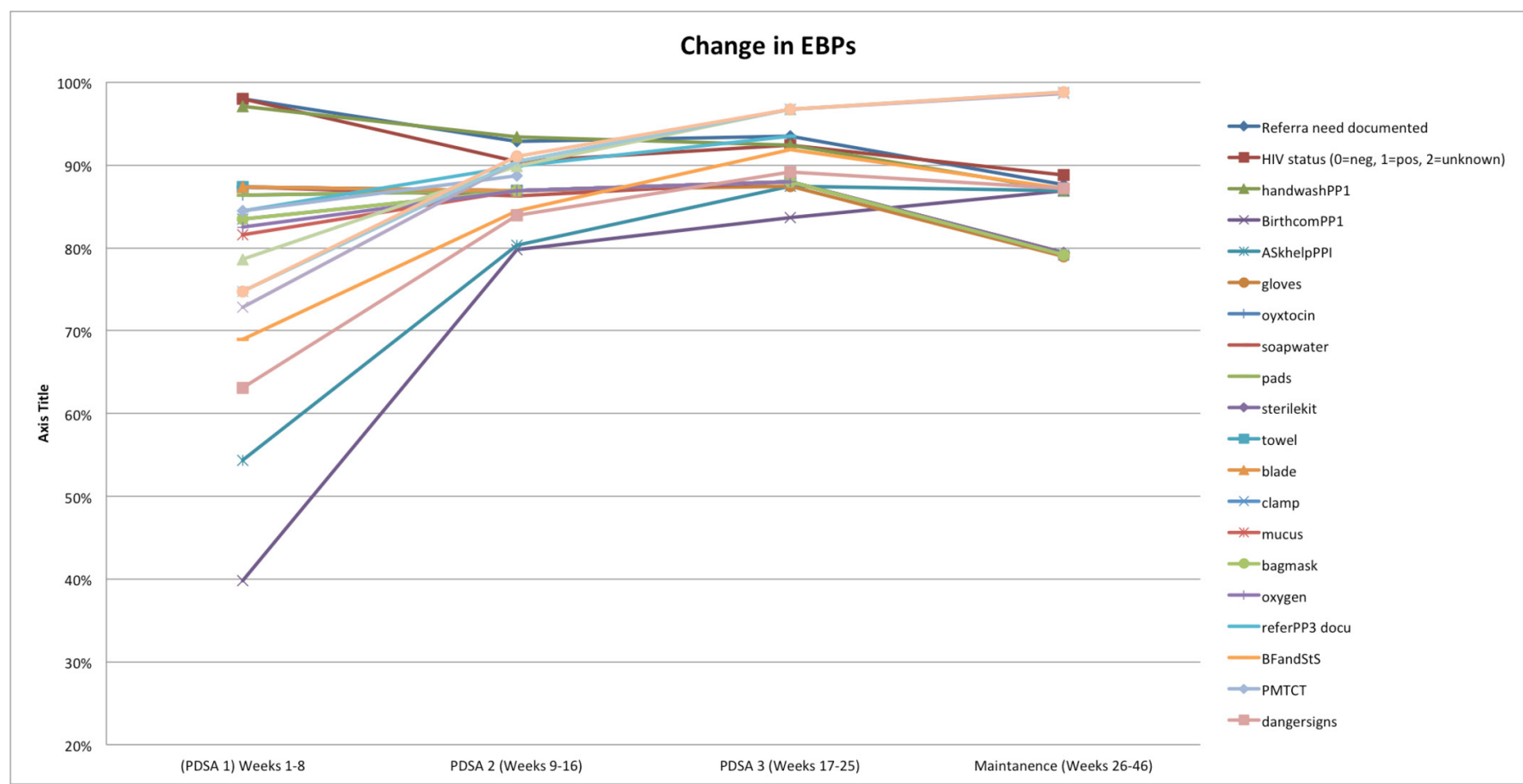

Figure 3 Average performance of each EBPs by PDSA cycle. EBPs, essential birth practices; PDSA, Plan-Do-Study-Act; PMTCT, prevention of mother-to-child transmission. 


\section{Study}

Initially, staff remained resistant to the 'paper burden' of the checklist, which was overcome as staff began to identify the utility of the checklist in reminding them of important steps during the birth process. Improvements in EBP adherence was observed, with an average of $88 \%$ of EBPs delivered and checklists used during $75 \%$ of deliveries (figures 1, 2 and 3).

Act

On reviewing the results, the remaining challenges identified included staff rotation resulting in new birth attendants unaware of the SCC intervention and unavailability of printers, especially during the night and weekend.

\section{PDSA cycle 3}

Plan and do

During the third PDSA cycle, changes to the intervention implementation included engaging management support to reduce staff rotation through the maternity ward. We focused on creating a committed and stable team sustaining the improvements in quality of maternity care. This was supported through the two quality champions and ongoing peer-to-peer coaching. The overall

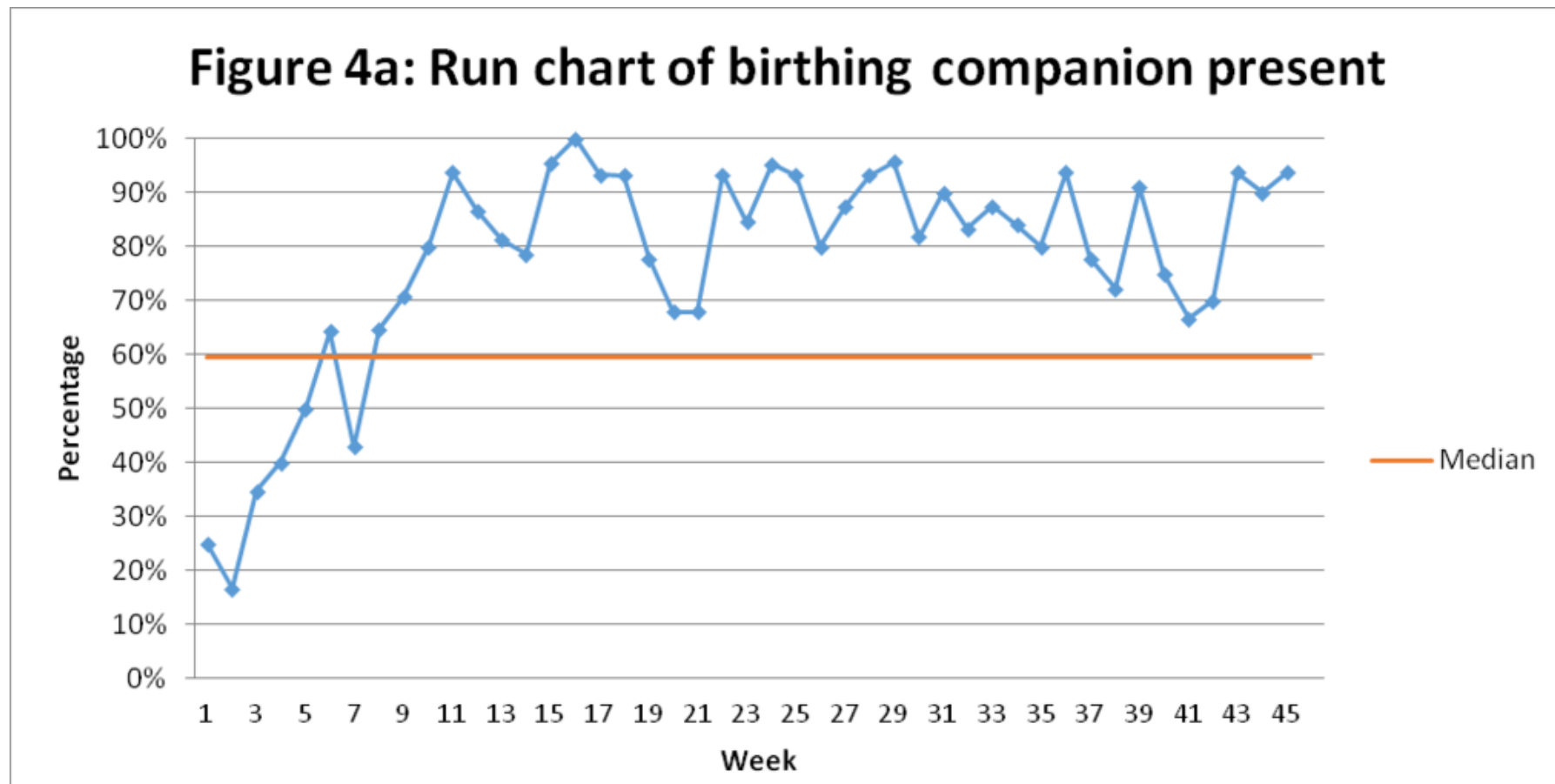

\section{Figure 4b: Run chart of immediate breast feeding}

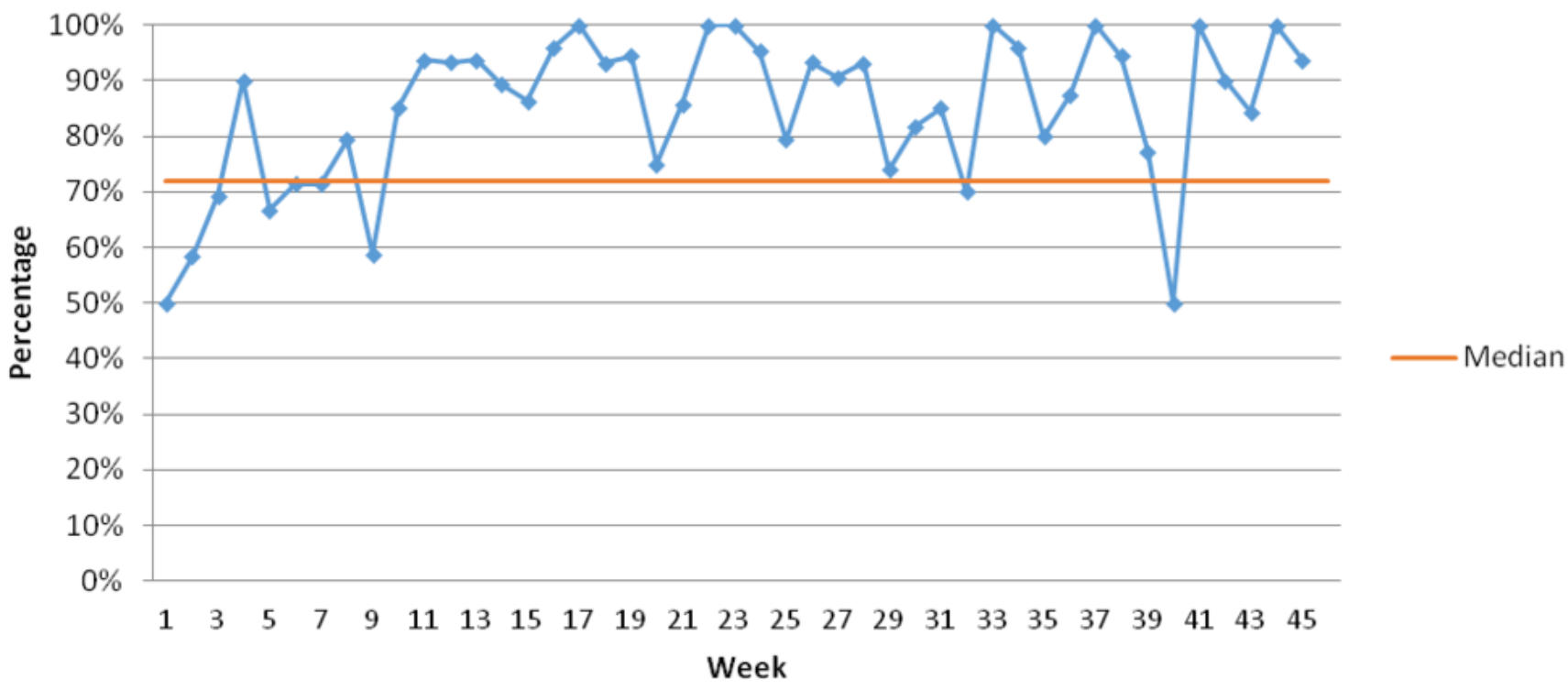

Figure 4 Run charts of behavioural change over the quality improvement initiative for (A) birth companion present at delivery and (B) immediate initiation of breast feeding in Gobabis District Hospital, Namibia. 


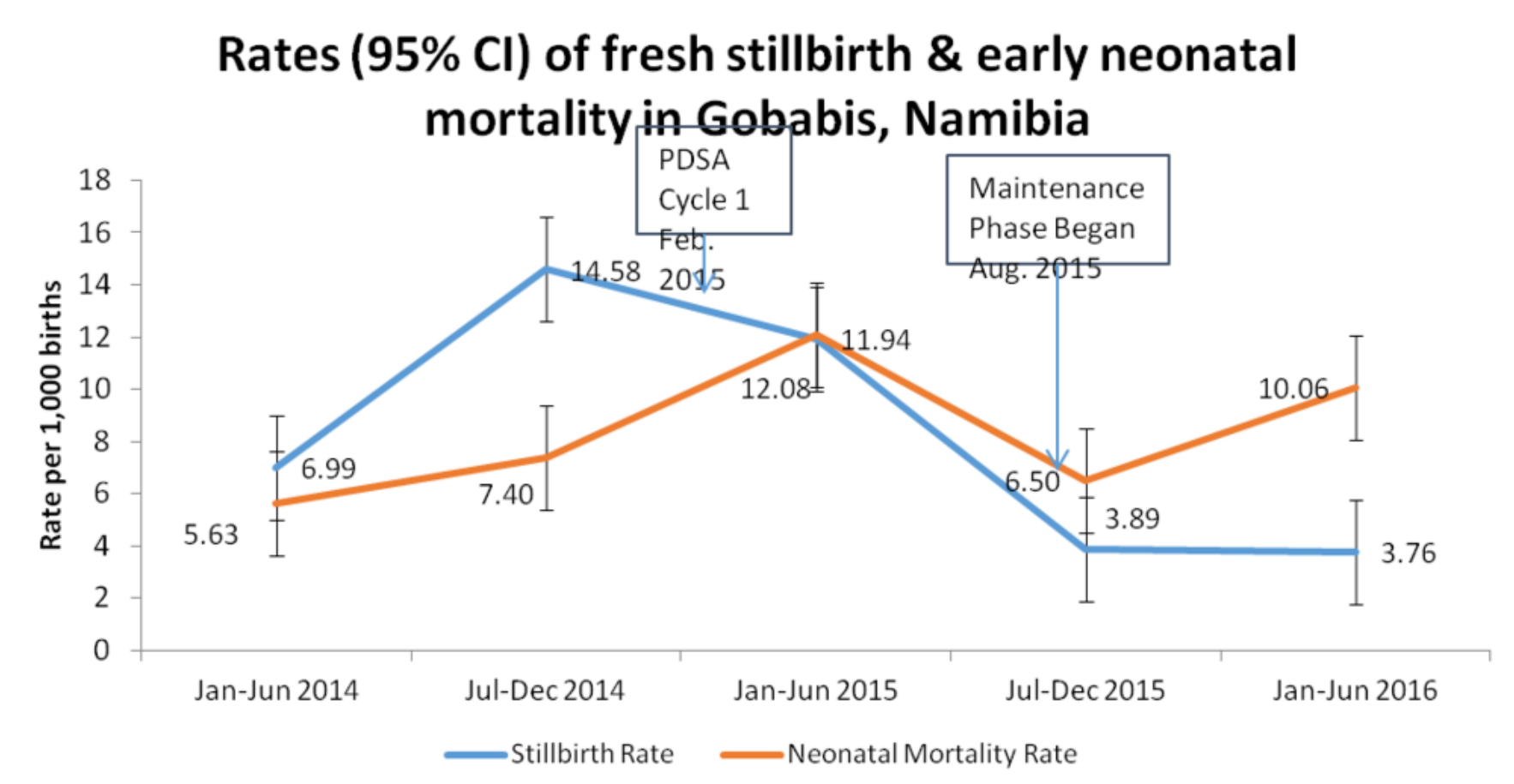

Figure 5 Change in fresh stillbirth and early in-hospital neonatal mortality rates. PDSA, Plan-Do-Study-Act.

aim was to create a task force of implementers that can use the tool with the sense of ownership.

\section{Study and act}

From weeks 17 to 24, WHO SCG use continued to increase with an average of $87 \%$ of deliveries having a checklist used. EBP adherence remained high, with an average of $93 \%$ of EBPs delivered (figures 2 and 3 ). At this point, the WHO SCC-based intervention was integrated into routine practice on the maternity ward (figure 1).

\section{Maintenance phase}

After the third PDSA cycle, the team continued with coaching and tracking EBPs and checklist use. The overall average rate of EBPs remained stable (88\% during the maintenance phase) with maintenance of some EBPs, which started lower than other changes and reached success by run chart analysis (figures 2, 3 and 4). There was some decline in selected EBPs, including availability of supplies at the bedside (figure 3). Ongoing coaching and leadership support was required. For example, there

Table 1 Gobabis District Hospital in-hospital FSB and early neonatal mortality*

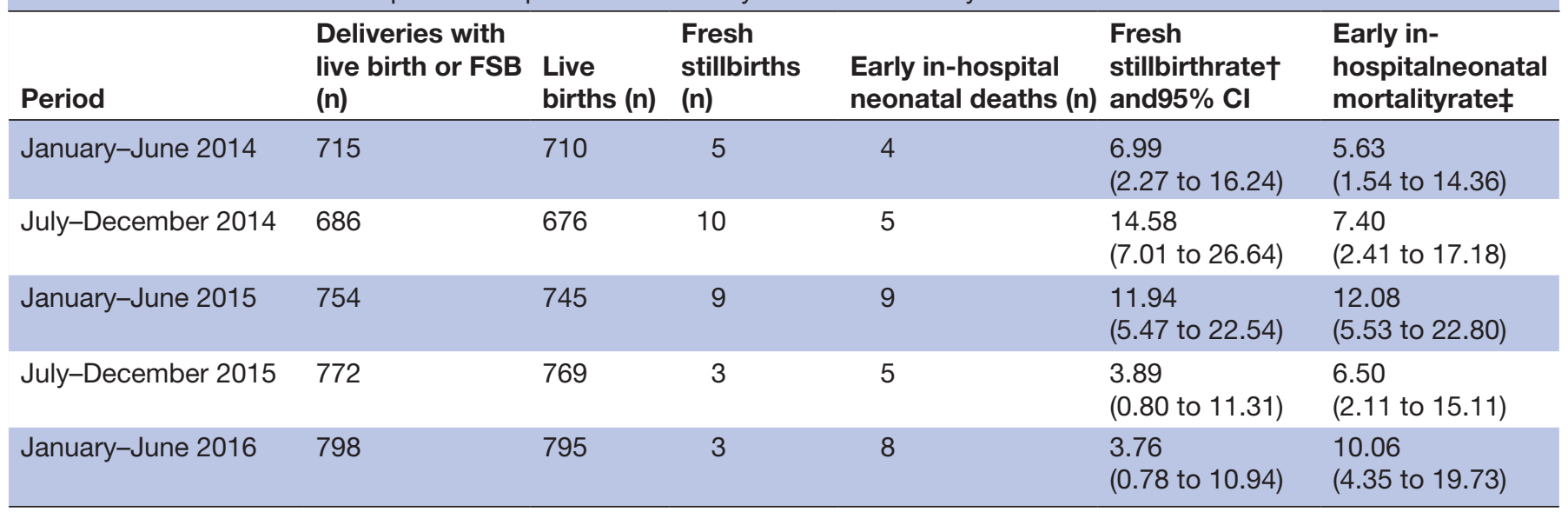

Fresh stillbirths' numbers excluded mothers admitted to the maternity ward with no fetal heart rate on admission and stillbirths determined to be macerated by the physicians.

${ }^{*}$ QI initiative started February 2015.

†Per 1000 live births+FSB.

†Per 1000 live births.

FSB, fresh stillbirth; QI, quality improvement. 
were 2 weeks where checklist use dropped because of decisions by nursing management around staff rotation in the case of new staff and loss of one of the nurse champions. However, this was rapidly identified by leadership; the staff that inappropriately rotated were brought back to the maternity ward. Thus, there was increased adherence to the EBP with return of the trained nurses and increase in coaching with improved of the checklist use (figure 1).

\section{Mortality rates}

Rates of fresh stillbirth remained relatively unchanged during the first 6 months of the intervention (14.58 to $11.94 / 1000$ live births + fresh stillbirths) and then dropped to $3.76 / 1000$, which was significantly lower than the pre-QI rates (table 1,Figure 5). Neonatal mortality rose during the first 6 months from 7.40 to 12.08 and then dropped to 6.50 , with an insignificant increase above the baseline up to 10.06 per a thousand during the maintenance phase. Overall perinatal mortality rates have almost halved from 22/1000 live births in the preintervention phase to 13.8 after three PDSA cycles largely because of the drop in fresh stillbirths(Figure 5).

\section{Lessons and limitations}

We found that implementing the WHO SCC using PDSA cycles developed an approach reflecting local context, which resulted in improvement in EBP adherence and fresh stillbirth rates but not in-hospital neonatal deaths. Key components included leadership support, SCC adaptation and peer-to-peer coaching to support implementation of the checklist. Implementation outcomes included feasibility and acceptability reflected by the increasing adoption of the SCC, as well as fidelity to the model of coaching to support SCC use and change birth attendant behaviour. ${ }^{12}$ In addition, evidence of effectiveness of the intervention was seen on the increased EBP adherence and declines in perinatal mortality driven by the drop in fresh stillbirths.

There were a number of components that were based on existing evidence of changes needed to drive behavioural change. ${ }^{13} 14$ These included an existing culture open to and experienced in organisational change and improvement, and a strong and committed leadership to provide motivation and facilitate changes in systems such as the staff rotations. The use of coaches to support adoption of interventions designed to increase adherence through behavioural change is also supported through the literature. The adaptation of using peers and facility champions trained in coaching also increased the coaching effectiveness. ${ }^{15} 16$ In addition, reflecting existing evidence, data feedback to the front-line providers associated with action planning also contributed to supporting individual and system behavioural change. The potential for sustainability (also a key implementation outcome) was made more likely by the use of existing resources and staff and integration into existing systems from the start.
There were a number of important organisational factors that likely contributed to the success. These included both existing resources, including an adequate nurse to patient ratio in the maternity unit and pre-existing QI experience and leadership at the facility (related to a national HIV improvement initiative ${ }^{17}$ ). In addition, the hospital leadership recognised that initial skills building was needed and addressed this gap through the neonatal resuscitation training conducted at the start of the project.

Our report has a number of limitations. First, we did not have counterfactual data to exclude external factors, which may have contributed to the observed changes. However, the leaders of the project were knowledgeable about the district and did not identify other interventions that may have resulted in the changes in quality or outcomes. Second, we relied on documented care, which can result in overestimation or underestimation of the quality as some of the changes may have been related to change in documentation rather than care. We also relied on the site physicians to distinguish between fresh and macerated stillbirths. We also only included hospital-based deaths, as we did not have the system and resources for follow-up for out-of-hospital early (within 7 days) or full neonatal mortality (within 28 days of delivery). We also did not have autopsies to determine cause of death for the early neonatal deaths, and so the understanding of why these rates did not drop as much as stillbirths was unknown. Finally, we were not able to capture the quality of care in deliveries where the SCC was either not used or not located.

\section{CONCLUSION}

In conclusion, we found that integrating a WHO SCC coaching intervention with strong leadership support and adherence to QI principles of rapid cycles of change was successful in improving the delivery of EBPs and was associated with some decline in perinatal mortality largely due to drops in fresh stillbirths. Scale-up of this SCC-based programme to all facilities in the Gobabis district is planned. The model was presented at the National Quality Management Unit and proposed for a rollout in all district hospitals in Namibia. Understanding the feasibility of replication of the intervention in other facilities using principles of QI and supporting the development of local champions will be important for effectiveness and long-term sustainability.

Acknowledgements The authors acknowledge the maternity team at Gobabis Hospital: Sr Moetsabatho, Sr Geingos, Sr Mbatara, Sr Chibaya, Sr Enkali, Sr Nambonga, Sr Makumbi, Sr Kananguizi, Dr Lumbala, Dr Shekimweri, Dr Ikandi, Sr Haingero, Sr Kazongominja and Ms Visajie, as well as the Namibia National Quality Assurance team: Dr Basenero, Dr Bruce Agins and the Informatics and Measurement Team at Ariadne Labs.

Funding This work was supported in part by a grant from the John D. and Catherine T. MacArthur Foundation.

Competing interests None declared.

Ethics approval Ethical approval was granted by the Namibia National Quality Assurance Unit, the agency coordinating all quality improvement projects in Namibia. 
No identifiable information was gathered and only data routinely collected as part of the quality improvement work was used.

Provenance and peer review Not commissioned; internally peer reviewed.

Open Access This is an Open Access article distributed in accordance with the Creative Commons Attribution Non Commercial (CC BY-NC 4.0) license, which permits others to distribute, remix, adapt, build upon this work non-commercially, and license their derivative works on different terms, provided the original work is properly cited and the use is non-commercial. See: http://creativecommons.org/ licenses/by-nc/4.0/

Published by the BMJ Publishing Group Limited. For permission to use (where not already granted under a licence) please go to http://www.bmj.com/company/ products-services/rights-and-licensing/

\section{REFERENCES}

1. Ministry of Health and Social Services Namibia. Namibia demographic and Health Survey 2013. Windhoek, Namibia: MoHSS/ Namibia and ICF International, 2014.

2. Ministry of Health and Social Services Namibia. Omaheke Regional Directorate Annual Report. Gobabis, Namibia, 2014.

3 Indongo N. Risk factors and causes of neonatal deaths in Namibia. European Scientific J [online] 2014:466-71.

4. World Health Organization. Millennium Development Goals and Beyond 2015. United Nations, 2000.

5. World Health Organization. WHO Safe Childbirth Checklist. 2015 http://www.who.int/patientsafety/implementation/checklists/ childbirth/en/ (accessed 27 Feb 2017).

6 Spector JM, Lashoher A, Agrawal P, et al. Designing the WHO Safe Childbirth Checklist program to improve quality of care at childbirth. Int J Gynaecol Obstet 2013;122:164-8.
7 Spector JM, Agrawal P, Kodkany B, et al. Improving quality of care for maternal and newborn health: prospective pilot study of the WHO Safe Childbirth Checklist program. PLoS One 2012;7:e35151.

8 World Health Organization. WHO Safe Childbirth Checklist Implementation Guide: Improving the Quality of Facility-Based Delivery for Mothers and Newborns. Geneva, Switzerland: World Health Organization, 2015.

9. Patabendige M, Senanayake H. Implementation of the WHO Safe Childbirth Checklist program at a tertiary care setting in Sri Lanka: a developing country experience. BMC Pregnancy Childbirth 2015;15:12.

10 Kumar S, Yadav V, Balasubramaniam S, et al. Effectiveness of the WHO SCC on improving adherence to essential practices during childbirth, in resource constrained settings. BMC Pregnancy Childbirth 2016;16:345.

11 Hunter J. The Deming 101: Theory of Knowledge and the PDSA Improvement and Learning Cycle. The E. Edwards Deming Institute Blog, 2013.

12 Proctor E, Silmere H, Raghavan R, et al. Outcomes for implementation research: conceptual distinctions, measurement challenges, and research agenda. Adm Policy Ment Health 2011;38:65-76.

13 Michie S, van Stralen MM, West R. The behaviour change wheel: a new method for characterising and designing behaviour change interventions. Implement Sci 2011;6:42.

14 Rowe AK, de Savigny D, Lanata CF, et al. How can we achieve and maintain high-quality performance of health workers in low-resource settings? Lancet 2005;366:1026-35.

15 Ellinger AD, et al. Theoretical traditions and coaching genres. Advances in Developing Human Resources 2014;16:139-60.

16 Greenhalgh T, Robert G, Macfarlane F, et al. Diffusion of innovations in service organizations: systematic review and recommendations. Milbank Q 2004;82:581-629.

17 HealthQual International. A public health approach to quality management. http://www.healthqual.org/namibia (accessed $14 \mathrm{Mar}$ 2017). 